\title{
Gravitational wave damping of neutron star wobble
}

\author{
Curt Cutler* \\ Max-Planck-Institut für Gravitationsphysik, Albert-Einstein-Institut, Am Muehlenberg 1, D-14476 Golm bei Potsdam, Germany \\ David Ian Jones ${ }^{\dagger}$ \\ Faculty of Mathematical Studies, University of Southampton, Highfield, Southampton, United Kingdom \\ and Department of Physics and Astronomy, University of Wales, College of Cardiff, P.O. Box 913, Cardiff, United Kingdom
}

(Received 9 August 2000; published 8 December 2000)

\begin{abstract}
We calculate the effect of gravitational wave $(\mathrm{GW})$ back reaction on realistic neutron stars (NS's) undergoing torque-free precession. By "realistic"' we mean that the NS is treated as a mostly fluid body with an elastic crust, as opposed to a rigid body. We find that GW's damp NS wobble on a time scale $\tau_{\theta} \sim 2$ $\times 10^{5} \mathrm{yr}\left[10^{-7} /\left(\Delta I_{d} / I_{0}\right)\right]^{2}\left(\mathrm{kHz} / \nu_{s}\right)^{4}$, where $\nu_{s}$ is the spin frequency and $\Delta I_{d}$ is the piece of the NS's inertia tensor that "follows" the crust's principal axis (as opposed to its spin axis). We give two different derivations of this result: one based solely on energy and angular momentum balance, and another obtained by adding the Burke-Thorne radiation reaction force to the Newtonian equations of motion. This problem was treated long ago by Bertotti and Anile, but their claimed result is wrong. When we convert from their notation to ours, we find that their $\tau_{\theta}$ is too short by a factor of $\sim 10^{5}$ for the typical cases of interest and even has the wrong sign for $\Delta I_{d}$ negative. We show where their calculation went astray.
\end{abstract}

DOI: $10.1103 /$ PhysRevD.63.024002

PACS number(s): 04.30.Db

\section{INTRODUCTION}

This paper calculates the effect of gravitational wave $(\mathrm{GW})$ back reaction on the torque-free precession, or wobble, of realistic, spinning neutron stars (NS's). By "realistic'" we mean the NS is treated as a mostly fluid body with an elastic crust, as opposed to a rigid body. (However, we do not include any superfluid effects in our analysis.) Freely precessing neutron stars are a possible source for the laser interferometer GW detectors [Laser Interferometer Gravitational Wave Observatory (LIGO), VIRGO, and GEO under construction, TAMA already operational]; it is the prospect of gravitational wave astronomy that motivated our study. Also, the first clear observation of free precession in a pulsar signal was reported very recently [1], with a modulation period consistent with the free precession model outlined in this paper, making this investigation all the more timely.

The effect of GW back reaction on wobbling, axisymmetric rigid bodies was first derived 27 years ago in an impressively early calculation by Bertotti and Anile [2]. They found (correctly) that for rigid bodies, GW back reaction damps wobble on a time scale (for small wobble angle $\theta$ ) $\tau_{\theta}^{\text {rigid }}$ $=1.8 \times 10^{6} \mathrm{yr} \quad\left[10^{-7} /\left(\Delta I / I_{1}\right)\right]^{2}\left(\mathrm{kHz} / \nu_{S}\right)^{4}\left(10^{45} \mathrm{~g} \mathrm{~cm}^{2} / I_{1}\right)$, where $\nu_{s}$ is the spin frequency and $\Delta I=\left(I_{3}-I_{1}\right)$ (with $I_{1}$ $=I_{2} \neq I_{3}$ ).

In the same paper, Bertotti and Anile [2] went on to calculate the effect of GW back reaction on wobble for the more realistic case of an elastic NS. When cast into our notation, their claimed GW time scale is $5 I_{1} c^{5} /\left[2 G\left(2 \pi \nu_{s}\right)^{4} \Delta I_{\Omega} \Delta I_{d}\right]$, where $\Delta I_{\Omega}$ is the asymmetry in the moment of inertia due to centrifugal forces and $\Delta I_{d}$ is

\footnotetext{
*Email address: cutler@aei-potsdam.mpg.de

†Email address: dij@maths.soton.ac.uk
}

the asymmetry due to some other mechanism, such as strain in the solid crust. Taking $\Delta I_{\Omega}$ to be (roughly) the asymmetry expected for a rotating fluid according to $\Delta I_{\Omega} / I$ $\approx 0.3\left(\nu_{s} / \mathrm{kHz}\right)^{2}$, we would then have a damping time of merely $0.6 \mathrm{yr}\left(\mathrm{kHz} / \nu_{s}\right)^{6}\left[10^{-7} /\left(\Delta I_{d} / I\right)\right]\left(10^{45} \mathrm{~g} \mathrm{~cm}^{2} / I\right)$. Despite the fundamental beauty of this problem and its potential astrophysical significance, their remarkable claim-that in realistic NS's, GW damp wobble with amazing efficiencywas apparently little known. (A citation index search showed that Bertotti and Anile [2] had been referenced by other authors only four times in the last 27 years.)

We will show that the Bertotti-Anile result for elastic NS's is very wrong, however. For typical cases of interest, their GW time scale $\tau_{\theta}$ is too short by a factor of $\sim 10^{5}$. Moreover, their calculation even gives the wrong sign (exponential growth instead of damping) when $\Delta I_{d}$ is negative. ${ }^{1}$ In contrast, we find that GW always act to damp the wobble in realistic NS's, just as for rigid bodies. While in nature the typical case will be $\Delta I_{d}$ positive, $\Delta I_{d}<0$ can also occur in principle. We call attention to this case not because it is common, but because it highlights how much our result differs from Bertotti and Anile [2] and because, in fact, their implicit prediction of exponential wobble growth for this case provided our initial impetus to look more closely at this problem.

\footnotetext{
${ }^{1}$ Actually, Bertotti and Anile [2] never claim in words that they find unstable growth of the wobble angle when $\Delta I_{d}<0$, but that is what is found if one just takes their formulas and converts from their notation to ours, as above. Moreover, we have repeated their (flawed) calculation, including their one crucial error, and seen that it does lead to a prediction of exponential wobble growth for $\Delta I_{d}$ negative. The conversion from their notation to ours is simply $\left(\delta_{1} I-\delta_{2} I\right)\left(\cos ^{2} \gamma-\frac{1}{2} \sin ^{2} \gamma\right) \rightarrow \Delta I_{d}$ and $\delta_{2} I \rightarrow \Delta I_{\Omega}$.
} 
The organization of this paper is as follows. In Sec. II we derive the GW damping time scale for rigid-body wobble, using the mass quadrupole expressions for the energy and angular momentum radiated to infinity. (This derivation is actually Exercise 16.13 in the textbook by Shapiro and Teukolsky [3].) We give another derivation of $\tau_{\theta}$ in Sec. III, this time by adding the Burke-Thorne radiation reaction force directly to the Newtonian equations of motion. This latter approach was how Bertotti and Anile [2] first calculated (correctly) the GW damping time for wobbling, rigid bodies.

In Sec. IV we review standard material on the torque-free precession of elastic bodies, in the absence of viscous terms or GW back reaction. In Sec. V we derive the GW damping time scale $\tau_{\theta}$ in the elastic case, using energy and angular momentum balance. In Sec. VI we give a second derivation of $\tau_{\theta}$ in the elastic case, using the Burke-Thorne radiation reaction force to evolve the elastic body's free precession. This was also the strategy of Bertotti and Anile [2], and we show where they went wrong. Briefly, they did not realize that in addition to torquing the NS, the radiation reaction force also perturbs the NS's shape (in particular, its inertia tensor). When solving for the evolution of the wobble angle, we show that the "perturbed shape" term in the equations of motion almost entirely cancels the GW torque term that they do include. (Of course, by definition there is no "perturbed shape" term in the rigid-body case, which is probably why they forgot this term when adapting that calculation to the elastic case.) In Sec. VII we describe how to include the effects of a fluid core in the radiation reaction calculation. Finally, in Sec. VIII we conclude by commenting briefly on the astrophysical implications of our result.

We will work in cgs units.

\section{RADIATION REACTION FOR A RIGID BODY: ENERGY AND ANGULAR MOMENTUM BALANCE}

The derivation of the wobble damping rate for realistic NS's, using energy and angular momentum balance, is rather similar to the corresponding derivation for rigid bodies. Here we briefly review the solution to the rigid-body problem, as a warm-up for tackling the realistic case.

Consider an axisymmetric rigid body with principal axes $\hat{x}_{1}, \hat{x}_{2}, \hat{x}_{3}$ and principal moments of inertia $I_{1}=I_{2} \neq I_{3}$. Let the body have angular momentum $\mathbf{J}$, misaligned from $\hat{x}_{3}$. Define the wobble angle $\theta$ by $\mathbf{J} \cdot \hat{\mathbf{x}}_{3}=J \cos \theta$. It is a standard result from classical mechanics that (in the absence of external torques) the body axis $\hat{x}_{3}$ precesses around $\mathbf{J}$ with (inertial frame) precession frequency $\dot{\phi}=J / I_{1}$, with $\theta$ constant [4]. Together, the pair $(\theta, \dot{\phi})$ completely specify the free precession (modulo a trivial constant of integration specifying $\phi$ at $t=0)$. We wish to calculate the evolution of these two parameters using the time-averaged fluxes $(\dot{E}, \dot{J})$.

Straightforward application of the mass quadrupole formalism [6] gives

$$
\dot{E}=-\frac{2 G}{5 c^{5}} \dot{\phi}^{6}(\Delta I)^{2} \sin ^{2} \theta\left(\cos ^{2} \theta+16 \sin ^{2} \theta\right),
$$

where $\Delta I=I_{3}-I_{1}$ and

$$
\dot{J}=\dot{E} / \dot{\phi} .
$$

It follows from differentiation of $\dot{\phi}=J / I_{1}$ that

$$
\ddot{\phi}=-\frac{2 G}{5 c^{5}} \frac{\Delta I^{2}}{I_{1}} \dot{\phi}^{5} \sin ^{2} \theta\left(16 \sin ^{2} \theta+\cos ^{2} \theta\right) .
$$

To calculate the rate of change of the wobble angle, rearrange

$$
\frac{d E}{d t}=\left.\frac{\partial E}{\partial J}\right|_{\theta} \frac{d J}{d t}+\left.\frac{\partial E}{\partial \theta}\right|_{J} \frac{d \theta}{d t}
$$

to give

$$
\dot{\theta}=\frac{\dot{J}\left[\dot{\phi}-\left.\frac{\partial E}{\partial J}\right|_{\theta}\right]}{\left.\frac{\partial E}{\partial \theta}\right|_{J}},
$$

where Eq. (2.2) has been used. The energy of the body is simply its kinetic energy

$$
E=\frac{J^{2}}{2 I_{1}}\left[1-\cos ^{2} \theta \frac{\Delta I}{I_{3}}\right],
$$

and so

$$
\begin{aligned}
& \left.\frac{\partial E}{\partial J}\right|_{\theta}=\frac{J}{I_{1}}\left[1-\cos ^{2} \theta \frac{\Delta I}{I_{3}}\right], \\
& \left.\frac{\partial E}{\partial \theta}\right|_{J}=\frac{J^{2}}{I_{1}} \cos \theta \sin \theta \frac{\Delta I}{I_{3}} .
\end{aligned}
$$

This gives

$$
\dot{\theta}=-\frac{2 G}{5 c^{5}} \frac{\Delta I^{2}}{I_{1}} \dot{\phi}^{4} \cos \theta \sin \theta\left(16 \sin ^{2} \theta+\cos ^{2} \theta\right) .
$$

We can construct time scales on which the spin-down and alignment occur:

$$
\begin{aligned}
\tau_{\dot{\phi}}^{\mathrm{rigid}} & =-\frac{\dot{\phi}}{\ddot{\phi}}=\frac{5 c^{5}}{2 G} \frac{1}{\dot{\phi}^{4}} \frac{I_{1}}{\Delta I^{2}} \frac{1}{\sin ^{2} \theta\left(16 \sin ^{2} \theta+\cos ^{2} \theta\right)}, \\
\tau_{\theta}^{\mathrm{rigid}} & =-\frac{\sin \theta}{\frac{d}{d t} \sin \theta}=\frac{5 c^{5}}{2 G} \frac{1}{\dot{\phi}^{4}} \frac{I_{1}}{\Delta I^{2}} \frac{1}{\cos ^{2} \theta\left(16 \sin ^{2} \theta+\cos ^{2} \theta\right)} .
\end{aligned}
$$

The radiation reaction causes both $\dot{\phi}$ and $\sin \theta$ to decrease, regardless of whether the body is oblate or prolate. Note that in the limit of small wobble angle the inertial precession 
frequency remains almost constant $\left(\tau_{\dot{\phi}}^{\text {rigid }} \rightarrow \infty\right)$, while $\theta$ decreases exponentially on the time scale

$$
\tau_{\theta \ll 1}^{\text {rigid }}=\frac{5 c^{5}}{2 G} \frac{1}{\dot{\phi}^{4}} \frac{I_{1}}{\Delta I^{2}} .
$$

Parametrizing,

$$
\tau_{\theta}^{\mathrm{rigid}}=1.8 \times 10^{6} \mathrm{yr}\left(\frac{10^{-7}}{\Delta I / I_{1}}\right)^{2}\left(\frac{\mathrm{kHz}}{\nu_{s}}\right)^{4}\left(\frac{10^{45} \mathrm{~g} \mathrm{~cm}^{2}}{I_{1}}\right) .
$$

In the limit of vanishingly small wobble angle, the partial derivative on the left-hand side (LHS) of Eq. (2.7) becomes what we conventionally call the "spin frequency" $\Omega$ of the body [5]. Equation (2.5) then shows that $\dot{\theta}$ is proportional to the difference between the inertial precession frequency $\dot{\phi}$ and the spin frequency $\Omega$. This difference remains finite as $\theta \rightarrow 0$ according to $\dot{\phi}-\Omega=\left(\Delta I / I_{1}\right) \Omega\left[1+\mathcal{O}\left(\theta^{2}\right)\right]$. Thus for a prolate body $(\Delta I<0)$, such as an American football, the body precesses slower than it spins, while for an oblate body the inertial precession frequency is higher than the spin frequency. Since the denominator in Eq. (2.5) is also proportional to $\Delta I$, the wobble angle decreases regardless of the sign of this factor. This viewpoint will be useful when we consider the radiation reaction problem for an elastic body.

\section{RADIATION REACTION FOR RIGID BODIES: LOCAL FORCE}

We will now rederive the spin-down and alignment time scales by adding the Burke-Thorne local radiation reaction force to the equations of motion.

The Burke-Thorne radiation reaction potential at a point $x$ is given by [6]

$$
\Phi^{\mathrm{RR}}=\frac{G}{c^{5}} x^{a} x^{b} \frac{d^{5} \Psi_{a b}}{d t^{5}}
$$

where $\Psi_{a b}$ denotes the trace-reduced quadrupole moment tensor:

$$
t_{a b}=\int_{V} \rho\left(x_{a} x_{b}-\frac{1}{3} \delta_{a b} x^{2}\right) d V
$$

Note that this is related to the moment of inertia tensor according to

$$
I_{a b}=-I_{a b}-\frac{2}{3} \delta_{a b} \int_{V} \rho x^{2} d V
$$

with the result that

$$
\Delta I \equiv I_{3}-I_{1}=-\left(I_{3}-I_{1}\right) .
$$

The radiation reaction force (on a particle of unit mass) is $F_{a}^{\mathrm{RR}}=-\partial \Phi^{\mathrm{RR}} / \partial x^{a}$. The instantaneous (not time-averaged) torque on a body can easily be shown to be

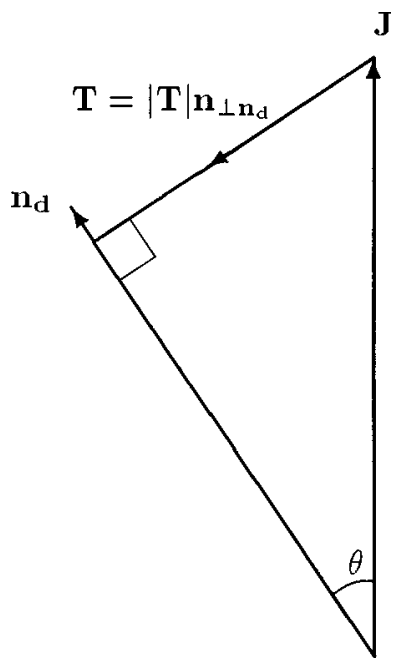

FIG. 1. For the rigid body the gravitational radiation reaction torque $\mathbf{T}$ lies in the reference plane. It acts perpendicular to the symmetry axis, i.e., along the direction of unit vector $\mathbf{n}_{\perp \mathbf{n}_{\mathbf{d}}}$.

$$
T^{a}=\frac{2 G}{5 c^{5}} \epsilon^{a b c} \Psi_{b d} \frac{d^{5} \Psi_{d c}}{d t^{5}} .
$$

Making use of Eq. (3.4) it is straightforward to calculate this torque for the free precessional motion. We find

$$
\mathbf{T}=\frac{2 G}{5 c^{5}} \Delta I^{2} \dot{\phi}^{5} \sin \theta\left(16 \sin ^{2} \theta+\cos ^{2} \theta\right) \mathbf{n}_{\perp \mathbf{n}_{\mathbf{d}}},
$$

acting always in the plane containing the angular momentum and the symmetry axis $x_{3}$, and perpendicular to $\mathbf{n}_{\mathbf{d}}$, i.e., along the direction of $\mathbf{n}_{\perp \mathbf{n}_{\mathbf{d}}}$ shown in Fig. 1. We will refer to this plane as the reference plane.

The evolution equations can be calculated without going to the trouble of writing down Euler's equations. Differentiation of $\dot{\phi}=J / I_{1}$ gives

$$
\ddot{\phi}=\frac{\dot{J}}{I_{1}}
$$

and so

$$
\ddot{\phi}=-\frac{T \sin \theta}{I_{1}} \text {. }
$$

Define $J_{\perp n_{d}}$ as the component of the angular momentum perpendicular to the symmetry axis. Then differentiation of the trivial relation

$$
\sin \theta=\frac{J_{\perp n_{d}}}{J}
$$

leads to

$$
\dot{\theta}=-\frac{T_{\perp J}}{J}=-\frac{T \cos \theta}{J},
$$


where $T_{\perp J}$ is the component of the torque perpendicular to $\mathbf{J}$. Equations (3.8) and (3.10) show that the action of the torque breaks down neatly into two parts. The component along $\mathbf{J}$ acts to change the inertial precession frequency $\dot{\phi}$, while the component perpendicular to $\mathbf{J}$ acts to change $\theta$. Substitution of Eq. (3.6) into Eqs. (3.8) and (3.10) then reproduces the spin-down and alignment of Eqs. (2.3) and (2.9), so the two methods of calculation agree. As this torque formulation makes clear [by combining Eqs. (3.8) and (3.10)], the product $\dot{\phi} \cos \theta$ remains constant, so that if a body is set into free precession described by $\left(\theta_{0}, \dot{\phi}_{0}\right)$, it tends to a nonprecessing motion about $x_{3}$ with (inertial frame) angular velocity $\dot{\phi}$ $=\cos \theta_{0} \dot{\phi}_{0}$.

\section{TORQUE-FREE PRECESSION OF ELASTIC BODIES}

We now review the theory of the free precession of an elastic body. This problem was first addressed in the context of the Earth's own motion. A rigorous treatment of the methods employed can be found in Munk and MacDonald [7]. The terrestrial analysis was extended to neutron stars by Pines and Shaham [8]. The energy loss due to gravitational waves was considered by Alpar and Pines [9].

Following the latter authors, we will model a star consisting of a centrifugal bulge and a single additional deformation bulge. Alpar and Pines wrote an inertia tensor for the elastic body of the form

$\mathbf{I}=I_{0, S} \boldsymbol{\delta}+\Delta I_{\Omega}\left(\mathbf{n}_{\Omega} \mathbf{n}_{\Omega}-1 / 3 \boldsymbol{\delta}\right)+\Delta I_{d}\left(\mathbf{n}_{\mathbf{d}} \mathbf{n}_{\mathbf{d}}-1 / 3 \boldsymbol{\delta}\right)$,

where $\boldsymbol{\delta}$ is the unit tensor $[1,1,1], \mathbf{n}_{\Omega}$ is the unit vector along the star's angular velocity $\boldsymbol{\Omega}$, and $\mathbf{n}_{\mathbf{d}}$ is the unit vector along the body's principal deformation axis (explained below). The $I_{0, S}$ and $\Delta I_{d}$ pieces of $\mathbf{I}$ together represent the inertia tensor for the corresponding nonrotating star. The $\Delta I_{d}$ term is just the nonspherical piece of this tensor (approximated as axisymmetric). If the star were a perfect fluid, $\Delta I_{d}$ would vanish, but in real stars (and the Earth) $\Delta I_{d}$ is nonzero due to crustal shear stresses and magnetic fields. The term $\Delta I_{\Omega}\left(>0\right.$ and $\propto \Omega^{2}$ for small $\left.\Omega\right)$ represents the increase in the star's moment of inertia (compared to the nonrotating case) due to centrifugal forces. Since the crust of a rotating NS will tend to "relax" towards its oblate shape, having $\Delta I_{d}>0$ is surely the typical case in nature. (E.g., if one could slow the Earth down to zero angular velocity without cracking its crust, it would remain somewhat oblate: the crust's "relaxed, zero-strain" shape is oblate, and after centrifugal forces are removed, the stresses that build up in the crust will act to push it back towards that relaxed shape.) But a negative $\Delta I_{d}$ is also possible in principle. We say the deformation bulge aligned with $\mathbf{n}_{\mathbf{d}}$ is "oblate" if $\Delta I_{d}>0$ and "prolate" if $\Delta I_{d}<0$.

What is a typical magnitude for $\Delta I_{d}$ in real, spinning NS's? Let us assume $\Delta I_{d}$ is due primarily to crustal shear stresses (as opposed to stresses in a hypothetical solid core, extremely strong $B$ fields, or pinned superfluid vortices). Then for a relaxed crust (i.e., a crust whose reference ellipticity is very close to its actual ellipticity), we have $\Delta I_{d}$ $=b \Delta I_{\Omega}$, where Alpar and Pines [9] estimate $b \sim 10^{-5}$ for a primordial (cold catalyzed) crust. The maximum value for $\Delta I_{d} / I$ is therefore of order $\sim 10^{-5}$. The parameter $b$ (which arises from internucleon Coulomb forces) scales like the average $Z^{2} / A$ of the crustal nuclei. Since crusts of accreted matter (as in LMXB's) have smaller- $Z$ nuclei [10], their $b$ factor is correspondingly smaller, by a factor of $\sim 2-3$. Using $\Delta I_{\Omega} / I \sim 0.3\left(\nu_{s} / \mathrm{kHz}\right)^{2}$, we would therefore estimate $\Delta I_{d} / I \sim 10^{-7}$ for a NS with a relaxed, accreted crust and $\nu_{s} \sim 300 \mathrm{~Hz}$, while for the Crab Nebula one would expect $\Delta I_{d} / I \sim 3 \times 10^{-9}$ (again, assuming its crust is almost relaxed). For the freely precessing pulsar reported in Stairs et al. [1], where the body-frame precession period is $\sim 2$ $\times 10^{8}$ times the rotation period, Eq. (4.15) below (valid for elastic bodies) yields $\Delta I_{d} / I=5 \times 10^{-9}$. For $b=10^{-5}$ this corresponds to a reference oblateness of $5 \times 10^{-4}$. This is consistent with the star's crust having solidified when it was spinning at about $40 \mathrm{~Hz}$, assuming that neither glitches nor plastic flow have modified its shape since. [When the effects of crust-core coupling are taken into account, giving Eq. (7.5), this initial frequency reduces to $12 \mathrm{~Hz}$. See Jones [11] for a review of pulsar free precession observations.]

Precession occurs when $\mathbf{n}_{\mathbf{d}}$ and $\mathbf{n}_{\boldsymbol{\Omega}}$ are not aligned. Below we describe the precessional motion when there is no damping. This analysis is quite general: it applies to any star whose inertia tensor is described by Eq. (4.1), independent of what causes the deformation bulge. In the case of several equally important sources of deformation along different axes, extra terms must be added to Eq. (4.1) and the analysis would become more complex.

To proceed it is necessary to use Eq. (4.1) to form the angular momentum $\mathbf{J}$ of the body. However, as we are not modeling a rigid body, we must take care to allow for the relative motion of one part with respect to another. Following [7] we will write the velocity of some point in the body as the sum of a rotational velocity with angular velocity $\boldsymbol{\Omega}$ and a small velocity $\mathbf{u}$ relative to this rotating frame. We will call the frame that rotates at $\boldsymbol{\Omega}$ the body frame, although it is only in the rigid-body limit that the body's shape is fixed with respect to this frame. In other words, the velocity of some particle making up the body is the sum of the bodyframe velocity $\boldsymbol{\Omega} \times \mathbf{r}$ at that point $\mathbf{r}$ plus the velocity $\mathbf{u}$ of the point relative to the body frame. Then

$$
J_{a}=I_{a b} \Omega_{b}+h_{a},
$$

where the possibly time-varying moment of inertia is defined in the usual way,

$$
I_{a b}=\int_{V} \rho\left(x_{c} x^{c} \delta_{a b}-x_{a} x_{b}\right) d V,
$$

while $h_{a}$ is the angular momentum of the body relative to this frame:

$$
h_{a}=\int_{V} \rho \epsilon_{a b c} x^{b} u^{c} d V .
$$



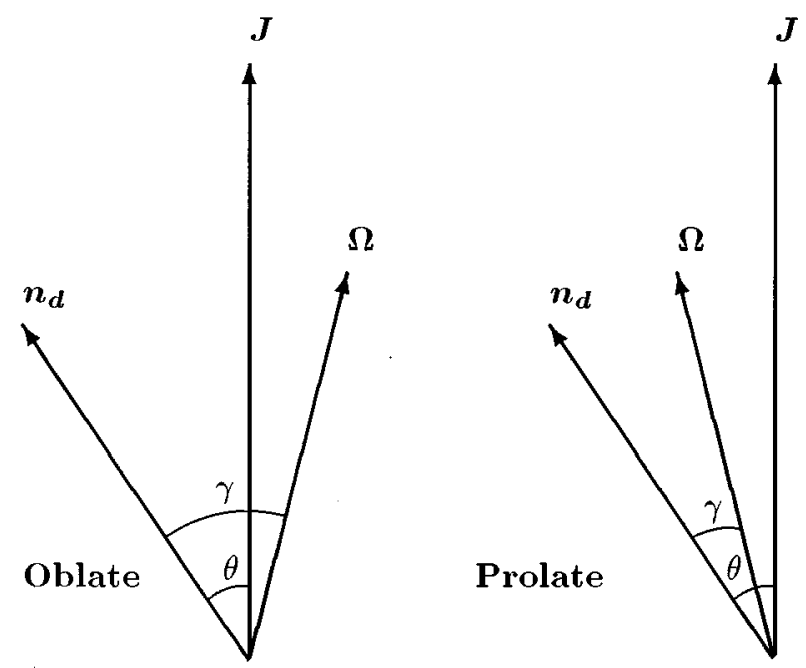

FIG. 2. This shows the reference plane, which contains the deformation axis $\mathbf{n}_{\mathbf{d}}$, the angular velocity vector $\boldsymbol{\Omega}$, and the fixed angular momentum $\mathbf{J}$. The vectors $n_{d}$ and $\Omega$ rotate around $\mathbf{J}$ at the inertial precession frequency $\dot{\phi}$. The terms "oblate" and "prolate" refer to the deformation bulge.

We will neglect the $h_{i}$ term when constructing a free precessional motion, as it can be shown that $h_{i}$ is small in a welldefined sense [11]. Therefore we will simply write

$$
J_{a}=I_{a b} \Omega^{b} .
$$

Having formulated the problem in this manner, it is straightforward to show that the free precession of an elastic body is similar to that of a rigid one. First, write down the angular momentum using Eqs. (4.1) and (4.5). Referring all of our tensors to the body frame, with the three-axis along $\mathbf{n}_{\mathbf{d}}$,

$$
\mathbf{J}=\left(I_{0, S}+2 / 3 \Delta I_{\Omega}-1 / 3 \Delta I_{d}\right) \boldsymbol{\Omega}+\Delta I_{d} \Omega_{3} \mathbf{n}_{\mathbf{d}} .
$$

This shows that $\mathbf{J}, \boldsymbol{\Omega}$, and $\mathbf{n}_{\mathbf{d}}$ are coplanar. As the angular momentum is constant, this plane must rotate about $\mathbf{J}$. As in the rigid-body case, we will refer to this as the reference plane. See Fig. 2. Taking the components of Eq. (4.6), we obtain

$$
\begin{aligned}
& J_{1}=\left(I_{0, S}+2 / 3 \Delta I_{\Omega}-1 / 3 \Delta I_{d}\right) \Omega_{1} \equiv I_{1} \Omega_{1}, \\
& J_{2}=\left(I_{0, S}+2 / 3 \Delta I_{\Omega}-1 / 3 \Delta I_{d}\right) \Omega_{2} \equiv I_{1} \Omega_{2}, \\
& J_{3}=\left(I_{0, S}+2 / 3 \Delta I_{\Omega}+2 / 3 \Delta I_{d}\right) \Omega_{3} \equiv I_{3} \Omega_{3} .
\end{aligned}
$$

These equations show that despite the triaxiality of $\mathbf{I}$ the angular momentum components themselves are structurally equivalent to those of a rigid symmetric top. The equations of motion of the body (i.e., Euler's equations) involve only the components of $\mathbf{J}$ and $\boldsymbol{\Omega}$. Therefore Eqs. (4.7)-(4.9) show that the free precession of the triaxial body is formally equivalent to that of a rigid symmetric top. We can think of the elastic body as having an effective moment of inertia tensor $\operatorname{diag}\left[I_{1}, I_{1}, I_{3}\right]$. Note that the effective oblateness $I_{3}$ $-I_{1}$ is equal to $\Delta I_{d}$.
Now introduce standard Euler angles to describe the body's orientation, with the polar axis along $\mathbf{J}$. Let $\theta$ and $\phi$ denote the polar and azimuthal coordinates of the deformation axis, while $\psi$ represents a rotation about this axis. We refer to $\theta$ as the wobble angle. Taking the ratio of components $J_{1}$ and $J_{3}$ using Eqs. (4.7) and (4.9) at an instant when $\Omega_{2}=0$, we obtain

$$
\tan \gamma=\frac{I_{3}}{I_{1}} \tan \theta,
$$

where $\gamma$ denotes the $\left(\boldsymbol{\Omega}, \mathbf{n}_{\mathbf{d}}\right)$ angle. See Fig. 2 .

We will label the angle between $\mathbf{J}$ and $\boldsymbol{\Omega}$ as $\hat{\theta}$ :

$$
\hat{\theta}=\gamma-\theta \text {. }
$$

This angle is much smaller than $\theta$, as can be seen by linearizing Eq. (4.10) in $\Delta I_{\Omega}$ and $\Delta I_{d}$ to give

$$
\hat{\theta}=\frac{\Delta I_{d}}{I_{3}} \sin \theta \cos \theta .
$$

Note that according to our conventions, when the deformation bulge is oblate, $\Delta I_{d}$ and $\hat{\theta}$ are positive, but when the deformation bulge is prolate, $\Delta I_{d}$ and $\hat{\theta}$ are negative.

We can decompose the angular velocity according to

$$
\boldsymbol{\Omega}=\dot{\phi} \mathbf{n}_{\mathbf{J}}+\dot{\psi} \mathbf{n}_{\mathbf{d}}
$$

Substituting this into Eq. (4.6) and resolving along $\mathbf{n}_{\mathbf{J}}$ and $\mathbf{n}_{\mathbf{d}}$ gives

$$
\begin{aligned}
& J=I_{1} \dot{\phi}, \\
& \dot{\psi}=-\frac{\Delta I_{d}}{I_{1}} \Omega_{3},
\end{aligned}
$$

where $J$ denotes the magnitude of the angular momentum. Note that when $\Delta I_{\Omega}=0$ the above formulas reduce to the familiar rigid-body equations.

Thus the motion is simple. As viewed from the inertial frame, the deformation axis rotates at a rate $\dot{\phi}$ in a cone of half-angle $\theta$ about the angular momentum vector. This angular velocity is sometimes called the inertial precession frequency. The centrifugal bulge rotates around the angular momentum vector also, but-for oblate deformations-on the opposite side of $\mathbf{J}$, making an angle $\hat{\theta} \equiv \gamma-\theta$ with $\mathbf{J}$. Superimposed upon this is a rotation about the deformation axis at a rate $\dot{\psi}$, known as the body-frame precession frequency or sometimes simply the precession frequency. This frequency is negative for an oblate distortion and positive for a prolate one.

\section{RADIATION REACTION FOR AN ELASTIC \\ BODY: ENERGY AND ANGULAR MOMENTUM BALANCE}

Here we derive the wobble damping time $\tau_{\theta}$ for elastic bodies, based on energy and angular momentum balance. 
Once fully underway, the derivation is just a couple lines. But to understand it, it is useful to carry along a simple, physical model for the deformed crust. (However, our derivation will actually be completely general.) Here is the model: take some nonrotating, spherical NS, and stretch a rubber band around some great circle on the crust. We shall refer to this great circle as the NS's equator. Obviously the effect of the rubber band is to make the NS slightly prolate (but still axisymmetric). To get an oblate shape, you can instead imagine sewing compressed springs into the surface of the crust at the equator. For definiteness, let the potential energy of the band (or springs) be $V=\frac{1}{2} \epsilon l^{2}$, where $l$ is its length. So $\epsilon$ is positive for the rubber band (prolate deformation, $\Delta I_{d}<0$ ) and negative for the springs (oblate deformation, $\Delta I_{d}>0$ ). Now give the NS angular momentum $\mathbf{J}$ about some axis that is not quite perpendicular to the equator. We now have our deformed, wobbling NS. We consider the equation of state of the star and the value $\epsilon$ to be fixed once and for all, and consider how the energy of the system (star+band) varies as a function of its total angular momentum $J$ and the wobble angle $\theta$ (the angle between $\mathbf{J}$ and the perpendicular to the equator); i.e., we consider $E(J, \theta)$. We will be concerned with small wobble angle, so let us expand $E(J, \theta)$ as a Taylor series in $J$ and $\theta$ :

$$
E(J, \theta)=E_{0}+\frac{1}{2} B J^{2}+\frac{1}{24} C J^{4}+\frac{1}{2} F \epsilon \theta^{2} J^{2}+\cdots .
$$

Here $E_{0}$ is defined to be the energy of the (star+band) at zero $J$, and $B, C$, and $F$ are some expansion coefficients that in principle depend on the physical properties of the (star+band). Fortunately, we will soon see that there are simple relations between $B, C$, and $F$ and previously defined physical parameters, such as $\Delta I_{d}$. Our ultimate goal is to obtain the two partial derivatives on the right-hand side of Eq. (2.5), where $E$ now denotes the total energy.

First, to see that no lower order terms (such as $J, \theta J, \theta^{2}$, or $\theta J^{2}$ terms) can appear in the expansion (5.1), note that the $J=0$ configuration corresponds to the minimum of the potential energy of the (star+band) system. Displacements of the (star+band) are first order in $J^{2}$, so changes in the potential energy of (star+band) are $\mathcal{O}\left(J^{4}\right)$. Thus terms in $E(J, \theta)$ that are $\propto J^{2}$ are kinetic energy pieces. These terms with a $J^{2}$ in them are clearly just $\frac{1}{2}\left(I_{0}^{-1}\right)^{a b} J_{a} J_{b}$, where $I_{0}^{a b}$ is defined to be the inertia tensor of the (star+band) at $J$ $=0$. [Corrections to the star's $I^{a b}$ first enter the energy at $\mathcal{O}\left(J^{4}\right)$.] We write $I_{0}^{a b}$ as

$$
I_{0}^{a b}=I_{0, S} \delta^{a b}+\Delta I_{d}\left(n_{d}^{a} n_{d}^{b}-\frac{1}{3} \delta^{a b}\right),
$$

where $I_{0, S}$ represents the "spherical part" of $I_{0}^{a b}$. Then

$$
\left(I_{0}^{-1}\right)^{a b}=\frac{1}{I_{0, S}}\left[\delta^{a b}-\left(\frac{\Delta I_{d}}{I_{0, S}}\right)\left(n_{d}^{a} n_{d}^{b}-\frac{1}{3} \delta^{a b}\right)\right],
$$

where a term of $\mathcal{O}\left(\Delta I_{d}^{2}\right)$ has been neglected. The kinetic energy part of $E$ is [up to terms of $\mathcal{O}\left(\Delta I_{d}^{2}\right)$ and $\mathcal{O}\left(J^{4}\right)$ ]

$$
E_{\mathrm{kin}}=\frac{J^{2}}{2 I_{0, S}}\left[1-\left(\frac{\Delta I_{d}}{I_{0, S}}\right)\left(\frac{2}{3}-\theta^{2}\right)\right],
$$

where we have used the small wobble angle result $J_{a} n_{d}^{a}$ $=J\left(1-\frac{1}{2} \theta^{2}\right)$. From Eq. (5.4) we immediately read off the values of $B$ and $F \epsilon$ in expansion (5.1):

$$
\begin{gathered}
B=I_{0, S}^{-1}\left[1-\frac{2}{3} \frac{\Delta I_{d}}{I_{0, S}}\right], \\
F \boldsymbol{\epsilon}=\Delta I_{d} /\left(I_{0, S}\right)^{2},
\end{gathered}
$$

and obtain the partial derivative

$$
\left.\frac{\partial E}{\partial \theta}\right|_{J}=J^{2} \theta \frac{\Delta I_{d}}{\left(I_{0, S}\right)^{2}} .
$$

To compute the partial derivative in the numerator of Eq. (2.5), it is sufficient to consider the $\theta \rightarrow 0$ limit [5] so that

$$
\Omega=\left.\frac{d E}{d J}\right|_{\theta=0}=B J+\frac{1}{6} C J^{3},
$$

where $\Omega$ denotes the spin frequency in the axisymmetric limit. It is related to the inertial precession frequency by

$$
\Omega=\dot{\phi}\left(1-\Delta I_{d} / I_{0, S}\right) .
$$

The final physics inputs we need are

$$
\begin{aligned}
& \dot{E}=-\frac{2 G}{5 c^{5}} \frac{\Delta I_{d}^{2}}{I_{0, S}} \dot{\phi}^{6} \theta^{2}, \\
& \dot{E}=\dot{\phi} \dot{J} .
\end{aligned}
$$

Equations (5.9) and (5.10) follow from the quadrupole formalism in the same way as for the rigid body.

The necessary pieces have been gathered; substituting into Eq. (2.5) gives

$$
\begin{aligned}
\dot{\theta} & =\frac{\dot{J} I_{0, S}^{2}}{\theta J^{2}} \frac{(\dot{\phi}-\Omega)}{\Delta I_{d}} \\
& =-\frac{2 G}{5 c^{5}}\left(\frac{\Delta I_{d}}{I_{0, S}}\right)^{2} I_{0, S} \dot{\phi}^{4} \theta .
\end{aligned}
$$

This is simply the same spin-down rate as for a rigid body, with the replacement $\left(\Delta I / I_{1}\right) \rightarrow \epsilon_{d}$. This is much longer than the time scale claimed by Bertotti and Anile [2] by a factor of $\Delta I_{\Omega} / \Delta I_{d}$, which is typically $\sim 10^{5}$ or higher.

Finally, the spin-down rate $\ddot{\phi}$ can be obtained in the same way as for a rigid body, i.e., by differentiating $\dot{\phi}=J / I_{1}$ and using Eqs. (5.9) and (5.10). Strictly, there will also be a term in $\dot{I}_{1}$, but this correction will be down by a factor of order $\left(\Omega / \Omega_{\max }\right)^{2}$. We then obtain the same spin down as for a rigid body, again with the replacement $\Delta I \rightarrow \Delta I_{d}$ : 


$$
\ddot{\phi}=-\frac{2 G}{5 c^{5}} \frac{\Delta I_{d}^{2}}{I_{0, S}} \dot{\phi}^{5} \theta^{2} .
$$

\section{RADIATION REACTION FOR AN ELASTIC BODY: LOCAL FORCE}

We now give a second derivation of the wobble damping rate for an elastic star, by directly adding the GW radiation reaction force to the Newtonian equations of motion. Besides being a satisfying consistency check on the calculation in Sec. IV, by doing this second derivation correctly we can show where Bertotti and Anile [2] went astray.

As was the case for the rigid body, the Burke-Thorne potential will exert a torque on the spinning star. However, this is not the only effect of the radiation reaction force: It will distort the shape of the NS and thus its moment of inertia. The equation describing the precession is then of the form

$$
\frac{d}{d t}\left[\left(\mathbf{I}_{N}+\delta \mathbf{I}_{\mathrm{BT}}\right) \boldsymbol{\Omega}\right]=\mathbf{T},
$$

where $\mathbf{I}_{N}$ denotes the Newtonian part of the moment of inertia tensor, $\delta \mathbf{I}_{\mathrm{BT}}$ the perturbation in this tensor due to the Burke-Thorne force, and $\mathbf{T}$ the Burke-Thorne torque. It was the $\delta \mathbf{I}_{\mathrm{BT}}$ terms that were not included by Bertotti and Anile. Fortunately, these can also be calculated explicitly, as we show below.

\section{A. Effect of $\Phi^{\mathrm{RR}}$ on the NS's shape}

It is perhaps surprising that one can explicitly determine the effect of $\Phi^{\mathrm{RR}}$ on the NS's moment of inertia, since the answer would seem to depend on the NS's mass and the details of its equation of state; i.e., one might worry that extra parameters must be specified even to make the problem well defined. However, the point is that (from symmetry arguments) the perturbation $\Delta I_{i j}$ depends only on a single physical parameter, and this parameter already appears in our Newtonian equations of motion. That parameter is $\Delta I_{\Omega} / \Omega^{2}$, the amount of oblateness caused "per unit centrifugal force."

The point is that both the centrifugal and radiation reaction forces have the very special property that they grow linearly with distance from the center of the star. This fact, coupled with symmetry arguments, is enough to determine $\Delta I_{i j}$ in terms of $\Delta I_{\Omega} / \Omega^{2}$; no new physical parameters have to be introduced.

Let $\Phi^{\Lambda}$ be some external potential of the form $\Phi^{\Lambda}$ $\equiv \Lambda^{a b} x_{a} x_{b}$, where $\Lambda_{a b}$ is some trace-free tensor. Allow this potential to act on the nonrotating (and so spherically symmetric) NS; it will induce a perturbation $\Delta I^{a b}$ in the NS's inertia tensor. Since the background is spherically symmetric, the only possibility (to first order in the perturbation) is that $\Delta I^{a b}=C \Lambda^{a b}$, where $C$ is some constant (i.e., independent of $\Lambda^{a b}$ ).

We can determine $C$ as follows. Decompose the centrifugal potential into a spherically symmetric and a trace-free piece:

$$
-\frac{1}{2} \Omega^{2}\left(\delta^{a b}-n_{\Omega}^{a} n_{\Omega}^{b}\right) x_{a} x_{b}=-\frac{1}{3} \Omega^{2} x^{2}+\Lambda_{\Omega}^{a b} x_{a} x_{b},
$$

where $\Lambda_{\Omega}^{a b}=\frac{1}{2} \Omega^{2}\left(n_{\Omega}^{a} n_{\Omega}^{b}-\frac{1}{3} \delta^{a b}\right)$. For small $\Omega$ the perturbed inertia tensor is $\Delta I^{a b}=\Delta I_{\Omega}\left(n_{\Omega}^{a} n_{\Omega}^{b}-\frac{1}{3} \delta^{a b}\right)$, so the constant $C$ is just $2 \Delta I_{\Omega} / \Omega^{2}$.

The radiation reaction potential for the freely precessing elastic body can be found by substituting the radiation reaction free motion into Eq. (3.1) to give

$$
\Phi^{\mathrm{RR}}=-\frac{G}{5 c^{5}} x^{a} x^{b}\left[\Delta I_{d} \frac{d^{5}}{d t^{5}}\left(\mathbf{n}_{\mathbf{d} a} \mathbf{n}_{\mathbf{d} b}\right)+\Delta I_{\Omega} \frac{d^{5}}{d t^{5}}\left(\mathbf{n}_{\mathbf{\Omega} a} \mathbf{n}_{\mathbf{\Omega} b}\right)\right] .
$$

The first term is the potential caused by the motion of the deformation bulge, the second by the centrifugal bulge. The differentiations of the unit vectors are straightforward. In the case where $\theta \ll 1$ we can approximate $\mathbf{n}_{\mathbf{d}} \approx \mathbf{n}_{\mathbf{J}}+\theta \mathbf{n}_{\perp \mathbf{J}}$ and $\mathbf{n}_{\mathbf{\Omega}} \approx \mathbf{n}_{\mathbf{J}}-\hat{\theta} \mathbf{n}_{\perp \mathbf{J}}$, where $\mathbf{n}_{\perp \mathbf{J}}$ is the unit vector in the reference plane which lies perpendicular to $\mathbf{J}$ and points towards $\mathbf{n}_{\mathbf{d}}$. We then find

$$
\Phi^{\mathrm{RR}}=-\frac{G}{5 c^{5}} \dot{\phi}^{5} x^{a} x^{b}\left[\Delta I_{d} \theta-\Delta I_{\Omega} \hat{\theta}\right]\left(\hat{\mathbf{v}}_{a} \mathbf{n}_{\mathbf{J} b}+\mathbf{n}_{\mathbf{J} a} \hat{\mathbf{v}}_{b}\right) .
$$

Here $\hat{\mathbf{v}}$ denotes a unit vector $\mathbf{n}_{\mathbf{J}} \times \mathbf{n}_{\perp \mathbf{J}}$. Using the prescription described above, these radiation reaction potentials can be converted immediately into perturbations of the moment of inertia tensor:

$$
\delta \mathbf{I}_{\mathrm{BT}}=-\frac{2 G}{5 c^{5}} \dot{\phi}^{3}\left[\Delta I_{d} \Delta I_{\Omega} \theta-\left(\Delta I_{\Omega}\right)^{2} \hat{\theta}\right]\left(\hat{\mathbf{v}} \mathbf{n}_{\mathbf{J}}+\mathbf{n}_{\mathbf{J}} \hat{\mathbf{v}}\right) .
$$

\section{B. Adding $\Phi^{R R}$ to the equations of motion}

It now remains to compute the torque $\mathbf{T}$ using Eq. (3.5). We obtain four terms, corresponding to the expansion of the product of $\boldsymbol{\Psi}$ with its fifth time derivative. Again linearizing with respect to $\theta$ we obtain

$$
\mathbf{T}=\frac{2 G}{5 c^{5}} \dot{\phi}^{5}\left[\Delta I_{d}^{2} \theta-\Delta I_{d} \Delta I_{\Omega} \hat{\theta}+\Delta I_{d} \Delta I_{\Omega} \theta-\Delta I_{\Omega}^{2} \hat{\theta}\right] \mathbf{n}_{\perp \mathbf{J}} .
$$

Define $\epsilon_{\Omega} \equiv \Delta I_{\Omega} / I_{0, S}$ and $\epsilon_{d} \equiv \Delta I_{d} / I_{0, S} .{ }^{2}$ Then the terms on the RHS of Eq. (6.6) stand in the ratio $\epsilon_{d} / \epsilon_{\Omega}: \epsilon_{d}: 1: \epsilon_{\Omega}$. We are now in a position to write down the equation for $d\left(\mathbf{I}_{N} \boldsymbol{\Omega}\right) / d t$. Using Eq. (6.5) and the Newtonian motion to compute $d\left[\left(\delta \mathbf{I}_{\mathrm{BT}}\right) \boldsymbol{\Omega}\right] / d t$ and neglecting terms of order $\theta^{2}$, we find that Eq. (6.1) reduces to

\footnotetext{
${ }^{2}$ Note our definition of $\epsilon_{\Omega}$ differs by a factor of $2 / 3$ from [9], who set $\epsilon_{\Omega} \equiv \frac{2}{3} \Delta I_{\Omega} / I_{0, S}$.
} 


$$
\begin{aligned}
& \frac{d}{d t}\left(\mathbf{I}_{N} \boldsymbol{\Omega}\right)+\frac{2 G}{5 c^{5}} \dot{\phi}^{5} \theta\left[\Delta I_{d} \Delta I_{\Omega} \theta-\left(\Delta I_{\Omega}\right)^{2} \hat{\theta}\right] \mathbf{n}_{\perp \mathbf{J}} \\
& \quad=\frac{2 G}{5 c^{5}} \dot{\phi}^{5}\left[\Delta I_{d}^{2} \theta-\Delta I_{d} \Delta I_{\Omega} \hat{\theta}+\Delta I_{d} \Delta I_{\Omega} \theta-\Delta I_{\Omega}^{2} \hat{\theta}\right] \mathbf{n}_{\perp \mathbf{J}}
\end{aligned}
$$

We see that the last two terms on the RHS are canceled by terms on the LHS. This leaves

$$
\frac{d}{d t}\left(\mathbf{I}_{N} \boldsymbol{\Omega}\right)=\frac{2 G}{5 c^{5}} \dot{\phi}^{5}\left[\Delta I_{d}^{2} \theta-\Delta I_{d} \Delta I_{\Omega} \hat{\theta}\right] \mathbf{n}_{\perp \mathbf{J}} .
$$

The problem is reduced to a rigid-body Newtonian one, with the two torque terms indicated on the right-hand side. The terms stand in the ratio $1: \epsilon_{\Omega}$. In fact, the dominant term is the same as that obtained in the rigid-body case with the change $\Delta I \rightarrow \Delta I_{d}$.

We therefore find that the alignment rate as calculated using the local Burke-Thorne formalism agrees with the flux-at-infinity method. The previous force-based calculation of Bertotti and Anile [2] failed to include the deformation $\delta \mathbf{I}_{\mathrm{BT}}$, so that the cancellations in Eq. (6.7) described above did not occur.

Finally, it is easy to show that even when the approximations $\theta \ll 1, \epsilon_{d} \ll 1$ are not employed, the effective torques due to the $\delta \mathbf{I}_{\mathrm{BT}}$ terms are still perpendicular to $\mathbf{J}$, so the spin-down $\ddot{\phi}$ using this local formalism is necessarily the same as in the flux-at-infinity method.

\section{ALLOWANCE FOR A LIQUID CORE}

We have successfully described the effects of gravitational radiation reaction on an elastic precessing body. We will now briefly describe how to extend this result to the realistic case where the star consists of an elastic shell (the crust) containing a liquid core. The Earth itself is just such a body, and the form of its free precession was considered long ago. We will base our treatment on that of Lamb [12], who considered a rigid shell containing an incompressible liquid of uniform density. To make the problem tractable the motion of the fluid was taken to be one of uniform vorticity. We will assume that the ellipticity of the shell and, also, the ellipticity of the cavity in which the fluid resides are small. Then the small-angle free precession of the combined system can be found by means of a normal mode analysis of the equations of motion [12].

The key points are as follows: The fluid's angular velocity vector does not significantly participate in the free precession. Instead, it remains pointing along the system's total angular momentum vector. The shell precesses about this axis in a cone of constant half-angle. The fluid exerts a force on the shell such that the shell's body frame precession frequency is increased in magnitude, so that

$$
\dot{\psi}=-\dot{\phi} \frac{\Delta I}{I_{\text {crust }}},
$$

where $\Delta I$ denotes the difference between the one and three principal moments of inertia of the whole body, not just the shell.

We now wish to calculate the alignment rate of such a body due to gravitational radiation reaction. The averaged energy and angular momentum fluxes, as well as the instantaneous torque, depend only upon the orientation of the mass quadrupole of the body and so are exactly the same as if the body were rigid: i.e., Eqs. (2.1), (2.2), and (3.6) apply. Equations giving the kinetic energy and angular momentum of the body are given in Lamb [12]. These can be used to obtain the partial derivatives that appear in Eq. (2.5). Explicitly, we find

$$
\left.\frac{\partial E}{\partial J}\right|_{\theta}=\Omega=\dot{\phi}+\dot{\psi}
$$

and

$$
\left.\frac{\partial E}{\partial \theta}\right|_{J}=\dot{\phi}^{2} \theta \Delta I
$$

(See Jones [11] for a detailed derivation.)

These lead to an alignment time scale that is $I_{\text {crust }} / I$ shorter than that of Eq. (2.13). This result is confirmed using the local torque formulation, where

$$
\dot{\theta}=-\frac{T_{\perp, J}}{I_{\text {crust }}} .
$$

In the realistic case where both crustal elasticity and core fluidity are taken into account, we can combine the above arguments as described by Smith and Dahlen [13]; i.e., we can take the rigid result and put $I \rightarrow I_{\text {crust }}$ and $\Delta I \rightarrow \Delta I_{d}$ to give

$$
\begin{aligned}
& \psi=-\dot{\phi} \frac{\Delta I_{d}}{I_{\text {crust }}}, \\
& \dot{\theta}=-\frac{2 G}{5 c^{5}} \frac{\Delta I_{d}^{2}}{I_{\text {crust }}} \dot{\phi}^{4} \theta .
\end{aligned}
$$

\section{CONCLUSIONS}

We have shown that the GW damping time for wobble in realistic NS's has the same form as for rigid bodies, but with the replacement $\Delta I^{2} / I_{1} \rightarrow \Delta I_{d}^{2} / I_{\text {crust }}$. This given an alignment time scale of

$$
\tau_{\theta}=1.8 \times 10^{5} \mathrm{yr}\left(\frac{I_{\text {crust }}}{10^{44} \mathrm{~g} \mathrm{~cm}^{2}}\right)\left(\frac{10^{38} \mathrm{~g} \mathrm{~cm}^{2}}{\Delta I_{d}}\right)^{2}\left(\frac{\mathrm{kHz}}{\nu_{s}}\right)^{4} .
$$

For the Crab Nebula, taking $\epsilon_{d} \sim 3 \times 10^{-9}$, this gives $\tau_{\theta} \sim 5$ $\times 10^{13} \mathrm{yr}-$ much longer than the age of the universe. For an accreting NS with $\epsilon_{d} \sim 10^{-7}$ and $\nu_{s} \sim 300 \mathrm{~Hz}$, we estimate $\tau_{\theta} \sim 2 \times 10^{8} \mathrm{yr}$.

Our basic conclusion, then, is that the GW back reaction is sufficiently weak that other sources of dissipation probably dominate. Unfortunately, even for the Earth the dissipa- 
tion mechanisms are not well understood [7]. Early estimates of Chau and Henriksen [14], which considered dissipation within the neutron star crust, suggested that wobble would be damped in around $10^{6}$ free precession periods, i.e., over a time interval of $10^{6} /\left(\epsilon_{d} \nu_{s}\right)$. A more recent study of Alpar and Sauls [15] argued that the dominant dissipation mechanism will be due to imperfect coupling between the crust and superfluid core. They estimate that the free precession will be damped in (at most) $10^{4}$ free precession periods. In contrast, according to Eq. (8.1), the GW damping time is in excess of $10^{8}\left(\mathrm{kHz} / \nu_{s}\right)^{3}$ free precession periods. On the basis of these estimates, it seems likely that internal damping will dominate over the gravitational radiation reaction in all neutron stars of interest. Note, however, that while internal dissipation damps wobble for oblate deformations, we expect that internal dissipation causes the wobble angle to increase in the prolate $\left(\Delta I_{d}<0\right)$ case.

A study of the gravitational wave detectability of realistic neutron stars undergoing free precession, including a discussion of other astrophysical mechanisms which might affect the evolution of the motion, will be presented elsewhere [16].

\section{ACKNOWLEDGMENTS}

We thank N. Andersson and B. F. Schutz for discussions. This research was supported by NASA grant NAG5-4093 and PPARC grant PPA/G/1998/00606.
[1] I. H. Stairs, A. G. Lyne, and S. L. Shemar, Nature (London) 406, 484 (2000).

[2] B. Bertotti and A. M. Anile, Astron. Astrophys. 28, 429 (1973).

[3] S. L. Shapiro and S. A. Teukolsky, Black Holes, White Dwarfs, and Neutron Stars (Wiley-Interscience, New York, 1983).

[4] L. D. Landau and E. M. Lifshitz, Mechanics, 3rd ed. (Butterworths-Heinemann, London, 1976).

[5] J. Ostriker and J. Gunn, Astrophys. J. 157, 1395 (1969).

[6] C. W. Misner, K. S. Thorne, and J. A. Wheeler, Gravitation (Freeman, San Francisco, 1973).

[7] W. H. Munk and G. J. F. MacDonald, The Rotation of the Earth (Cambridge University Press, Cambridge, England,
1960).

[8] D. Pines and J. Shaham, Nature (London), Phys. Sci. 235, 43 (1972); Phys. Earth Planet. Inter. 6, 103 (1972).

[9] A. Alpar and D. Pines, Nature (London) 314, 334 (1985).

[10] G. Ushomirsky, C. Cutler, and L. Bildsten, astro-ph/0001136.

[11] D. I. Jones, Ph.D. thesis, University of Wales, Cardiff, 2000.

[12] H. Lamb, Hydrodynamics, 6th ed. (Cambridge University Press, Cambridge, England, 1952).

[13] M. L. Smith and L. A. Dahlen, Geophys. J. R. Astron. Soc. 64, 223 (1981).

[14] W. Y. Chau and R. N. Henriksen, Astrophys. Lett. 8, 49 (1971).

[15] A. Alpar and J. A. Sauls, Astrophys. J. 327, 723 (1988).

[16] D. I. Jones, B. F. Schutz and N. Andersson (in preparation). 